\title{
In Vivo Detection of the Effect of Electroacupuncture on "Zusanli" Acupoint in Rats with Adjuvant-Induced Arthritis through Optical Coherence Tomography
}

\author{
Huiqing Zhong, ${ }^{1}$ Hui Yang, ${ }^{2}$ Yan Zhou, ${ }^{2}$ Zhouyi Guo, ${ }^{1}$ Xiuli Wu, ${ }^{1}$ Chengkang Su, \\ Jia Long, ${ }^{2}$ Jin Lin, ${ }^{2}$ and Xuemei Jiang ${ }^{1}$ \\ ${ }^{1}$ SATCM Third Grade Laboratory of Chinese Medicine and Photonics Technology, College of Biophotonics, \\ South China Normal University, Guangzhou 510631, China \\ ${ }^{2}$ Infinitus (China) Company Ltd., Guangzhou 510665, China \\ Correspondence should be addressed to Zhouyi Guo; ann@scnu.edu.cn
}

Received 24 June 2016; Accepted 20 October 2016

Academic Editor: Adair Santos

Copyright $\odot 2016$ Huiqing Zhong et al. This is an open access article distributed under the Creative Commons Attribution License, which permits unrestricted use, distribution, and reproduction in any medium, provided the original work is properly cited.

\begin{abstract}
This study aimed to investigate the effect of electroacupuncture (EA) treatment through optical coherence tomography (OCT) in vivo on rats with adjuvant-induced arthritis. OCT images were obtained from the ankle of the right hind paws of the rats in control, model, and EA groups before modelling and 1 day, 8 days, 15 days, 22 days, and 29 days after modelling. Results demonstrated that the OCT signal of the ankle of the right hind paws of the rats was indistinct compared to 1 day after modelling and before modelling in the EA group. In the EA group, the light averaged attenuation coefficients of the ankle tissues decreased as treatment duration was prolonged after EA was administered $\left(3.43,2.96,2.61,2.42\right.$, and $2.29 \mathrm{~mm}^{-1}$, resp.). There was a significant difference in attenuation coefficient decrease between the 29th $\mathrm{d}$ and the 1st $\mathrm{d}$ for EA group compared with control group $(P<0.01)$. This condition indicated that the light absorption of the ankle of the treated rats in the EA group decreased. Therefore, OCT can be used to monitor the effect of treatment on rats with arthritis in vivo.
\end{abstract}

\section{Introduction}

Complete Freund's adjuvant is used to induce inflammatory pain in animal models of diseases, such as arthritis. It is often utilised to investigate pain mechanisms [1-3], pain relief [4], and drug-induced anti-inflammatory effects and to explain physical acupuncture methods [5-10]. The effects of treatments on arthritis have been detected by direct observation through the eyes or by measuring circumference in vivo. However, these methods fail to detect the change in the depth of arthritis.

OCT is an optical technique used to determine the backscattering of near-infrared light in tissues. OCT is a recently developed noninvasive imaging technique that provides high-resolution cross-sectional images of biological tissue microstructures [11-13]. This technique combines the advantages of interferometers and confocal microscopes to probe weakly backscattered photons from microstructures beneath tissue surfaces. OCT has been successfully applied in vivo in medical imaging and diagnostics, such as ophthalmic $[14,15]$, dermatological [16-18], eye [19], glucose [20], and oral [21-25] treatments.

In this study, OCT was employed to detect the effect of electroacupuncture (EA) treatment on rats. This study aimed to monitor the in vivo effect of treatment on rats with arthritis through OCT.

\section{Materials and Methods}

2.1. Animals. Forty-five male and female Wistar rats aged 810 weeks and weighing $190 \pm 10 \mathrm{~g}$ were obtained from the Laboratory Animal Centre, Southern Medical University. All experiments were performed under protocols approved by the South China Normal University Animal Care and Use Committee. The rats were housed at a controlled temperature $\left(23 \pm 2^{\circ} \mathrm{C}\right)$, habituated ad libitum, and maintained at $12 \mathrm{~h} / 12 \mathrm{~h}$ 
light/dark cycle. All of the rats were habituated at the laboratory room for at least 1 week before the experiment began. They were then divided randomly into three groups: (1) control group $(n=15)$, which was not treated; (2) model group ( $n=15)$, which was not treated after the model was established; and (3) electroacupuncture group ( $n=15)$, which was treated with right lateral "Zusanli" electroacupuncture (ST36; ST36 acupoint is located between the tibia and the fibula [26]) after the model was established. Each animal in the model and electroacupuncture groups was injected with $0.1 \mathrm{~mL}$ of complete Freund's adjuvant (CFA, Sigma, USA) into the ankle joint of the right hind paw. The CFA injection immediately caused local inflammation, paw swelling, and pain. These symptoms indicated that the model was successfully established.

2.2. Electroacupuncture Treatment. From 1 day after modelling, stainless steel acupuncture needles with a diameter of $0.25 \mathrm{~mm}$ were inserted into the ST36 acupoint of the right hind paw ipsilaterally at a depth of $7 \mathrm{~mm}$. Continuous-wave stimulation was selected at a frequency of $5 \mathrm{~Hz}$ and intensity of 2 mA (6805-AII, Shantou Medical Equipment Factory Co., Ltd.) [27-29]. An individual EA session was administered daily for $20 \mathrm{~min}$ for 7 days, with 2-day rest for the course of treatment, for 4 consecutive weeks.

2.3. OCT System and Analysis of OCT Signal Attenuation. The OCT system used in this study mainly consisted of resource, fibre conduction, reference arm, and sample arm modules. A schematic of the OCT system is shown in a previous study [25]. The broadband light source was a superluminescent diode with a central wavelength of $1310 \mathrm{~nm}$ and a bandwidth of $50 \mathrm{~nm}$. The light source yields an axial resolution of $15 \mu \mathrm{m}$ in a free space. The transverse resolution of the system was approximately $20 \mu \mathrm{m}$, as determined by the focal spot size produced by the probe beam. The signal-to-noise ratio of this system was measured at $100 \mathrm{~dB}$. A visible light source $(\lambda=$ $632 \mathrm{~nm}$ ) was used to guide the probe beam. The OCT system operation was controlled automatically by using a computer $[25,30,31]$. The right ankle joints of the rats in each group were monitored at different time points $(0,1,8,15,22$, and 29 days) through OCT.

OCT imaging is based on the difference in the backscattering of light. In this model, light that scattered with the decay of the OCT signal with a depth function is in accordance with Beer-Lambert law. According to Beer-Lambert law, light attenuation inside tissues is exponential. The total attenuation coefficient is $\mu_{t}=\mu_{s}+\mu_{a}$, where $\mu_{s}$ is the scattering coefficient and $\mu_{a}$ is the absorption coefficient. Only the backscattered components from tissues contribute to the OCT image because $\mu_{a} \ll \mu_{s}$ for tissues in the NIR spectral range [32-34]. In brief, the differences in the attenuation coefficient in each group can potentially be detected with the OCT system from $\mu_{t}$. The following expression is a Liebenberg-Marquardt curve fitting algorithm [25, 34]:

$$
y=A \exp \left(-\mu_{\mathrm{t}} x\right)+y_{0}
$$

where $y$ is the signal, $x$ is the penetration depth of the OCT images, and $\mu_{t}$ is the attenuation coefficient.
2.4. Statistical Analysis. Data were presented as means $\pm \mathrm{SD}$ for a number of sample animals and analysed through paired $t$-test by using SPSS 18.0. $P<0.05$ indicated significant difference.

\section{Results and Discussion}

3.1. Photos. The rats in the control group were not treated, in which no change was observed in the hind paw (not shown). The rats' hind paws in the model group before modelling, 1 day after modelling, 8 days after modelling, 15 days after modelling, 22 days after modelling, and 29 days after modeling are shown in Figure 1. Comparing Figure 1(b) with Figure 1(a), we found that the swelling in the right hind paw was different. By contrast, this finding is not observed in the control group. This result implied that the model was successfully established. After the model was created, the right hind paw could slowly self-heal. However, the effect was not distinct. In some instances, the swelling in the right hind paw may become serious (comparing Figure 1(e) with Figure $1(\mathrm{~d}))$. The rats' hind paws in the electroacupuncture group before modelling, 1 day after modelling, 8 days after modelling, 15 days after modelling, 22 days after modelling, and 29 days after modeling are shown in Figure 2. From 1 day after modelling, the rats in the electroacupuncture group were treated with electroacupuncture for $20 \mathrm{~min}$. It was observed that the swelling in the right hind paw was different (Figures 2(b) and 2(a)). After the treatment time was prolonged, the swelling in the right hind paws of the rats was relieved. On day 29, the swelling in the right hind paws of the rats almost disappeared (Figure 2(f)). This condition indicated that electroacupuncture could stimulate the "Zusanli" acupoint to treat the adjuvant-induced arthritis.

3.2. Analysis of OCT Signal Attenuation. From the OCT images of the ankle of the right hind paws of the rats in the control group at different times in vivo, it can be found that their surface layers of the normal ankle of the right hind paws of the rats are visible with a quite distinct layer structure (not shown).

However, the layer is difficult to be distinguished because it is injected with $0.1 \mathrm{~mL}$ complete Freund's adjuvant in the ankle joint of the right hind paw with swelling (Figure 3(b)). Thus, the OCT signal of the ankle of the right hind paws of the rats shows significant attenuation. This result is in accordance with Figure 1(b). This condition confirms that complete Freund's adjuvant can decrease the OCT signal of local tissues when the adjuvant is locally injected in tissues. This decrease occurs because the rats were not treated in every way after the model was established. Thus, the OCT signals in the ankle of the right hind paws of the rats are also structurally indistinct when the treatment time was prolonged after the model was established (Figures 3(b)-3(f)). The OCT images of the ankle of the right hind paws of the rats in the electroacupuncture group before modelling, 1 day after modelling, 8 days after modelling, 15 days after modelling, 22 days after modelling, and 29 days after modelling are shown in Figure 4. In Figure 4(b), the OCT signal of the ankle of the right hind paws of the rats is indistinct. However, 


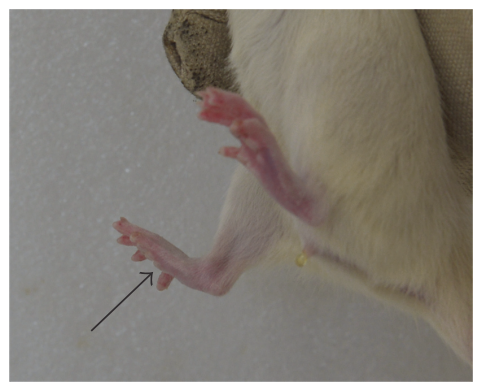

(a)

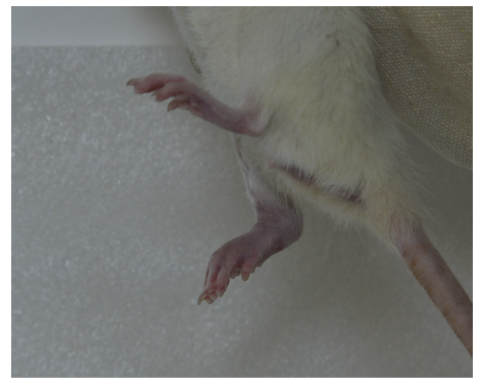

(d)

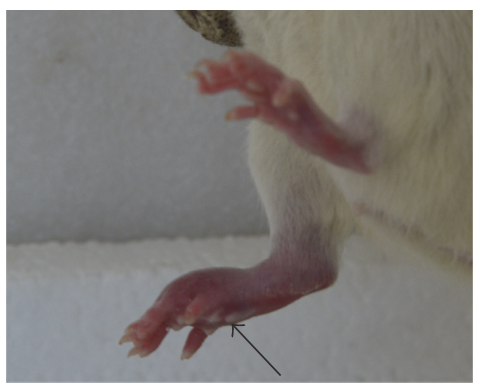

(b)

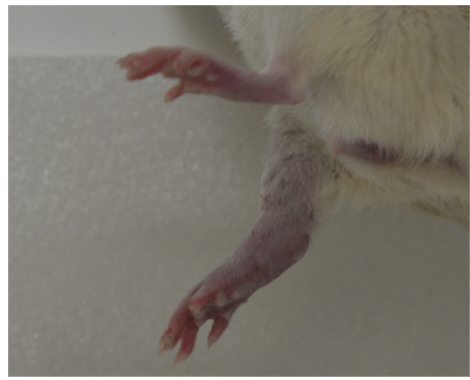

(e)

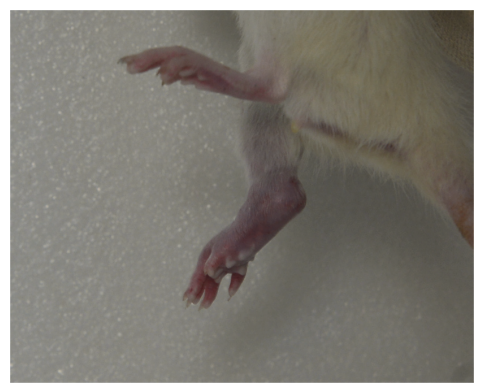

(c)

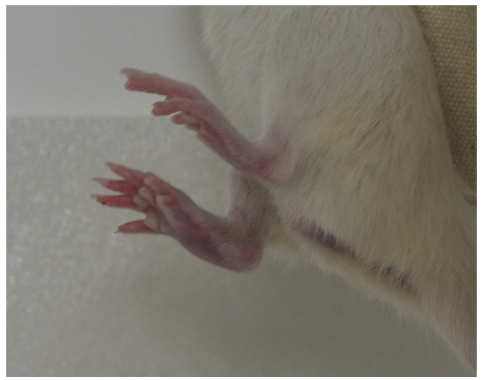

(f)

FIGURE 1: Representative photos of paws of mice in the model group before modelling (a), 1 day after modelling (b), 8 days after modelling (c), 15 days after modelling (d), 22 days after modelling (e), and 29 days after modelling (f). The black arrow is the location of injecting complete Freund's adjuvant.

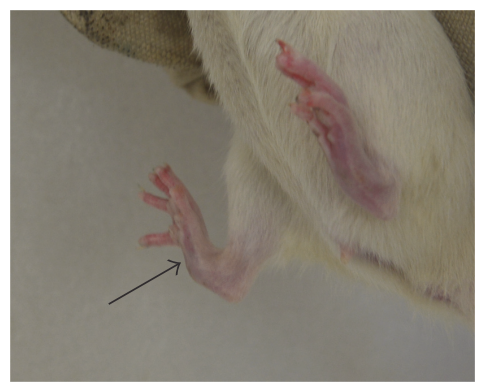

(a)

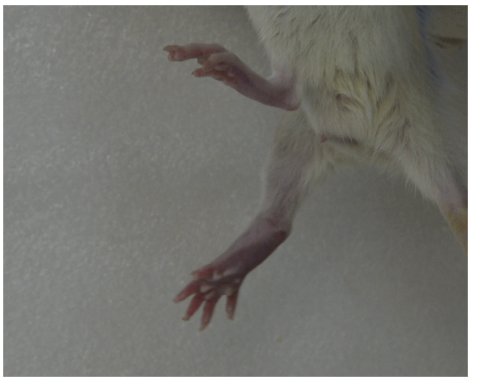

(d)

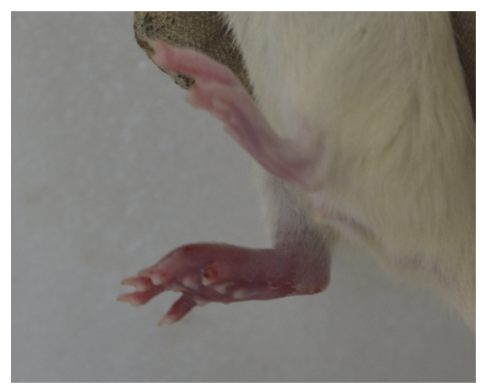

(b)

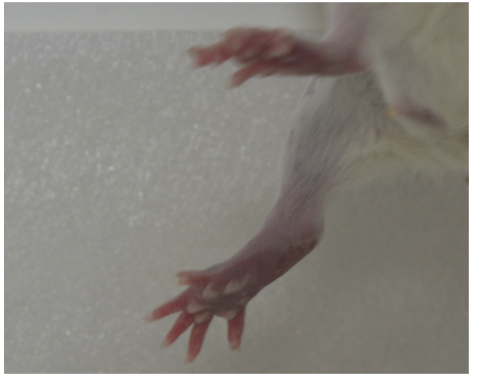

(e)

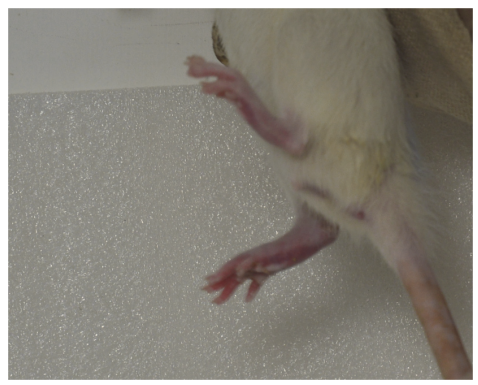

(c)

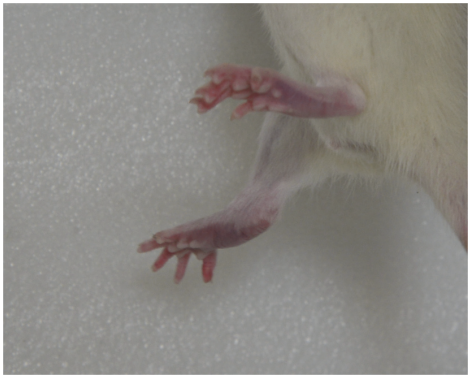

(f)

FIGURE 2: Representative photos of paws of mice in the electroacupuncture group before modelling (a), 1 day after modelling (b), 8 days after modelling (c), 15 days after modelling (d), 22 days after modelling (e), and 29 days after modelling (f). The black arrow is the location of injecting complete Freund's adjuvant. 


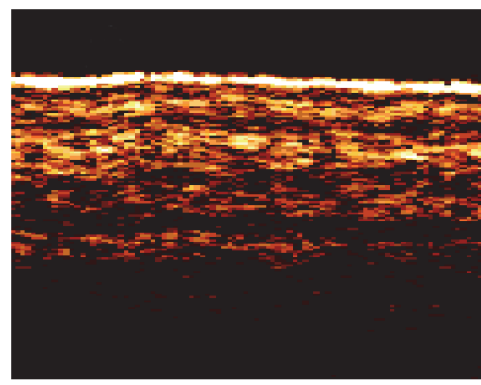

(a)

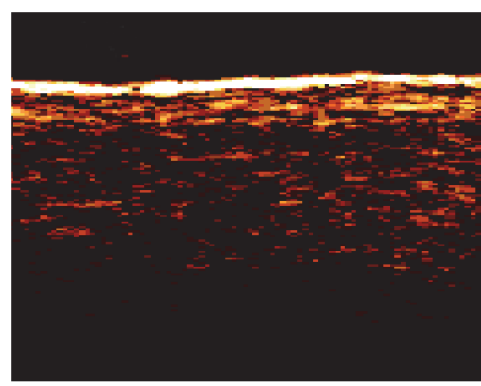

(d)

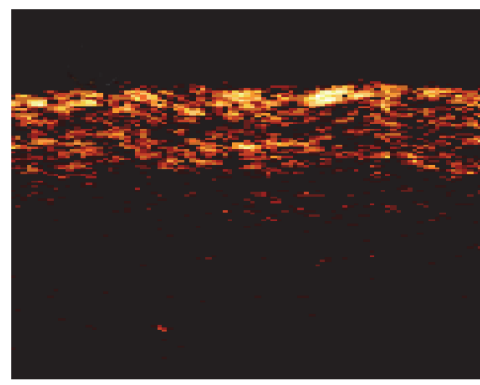

(b)

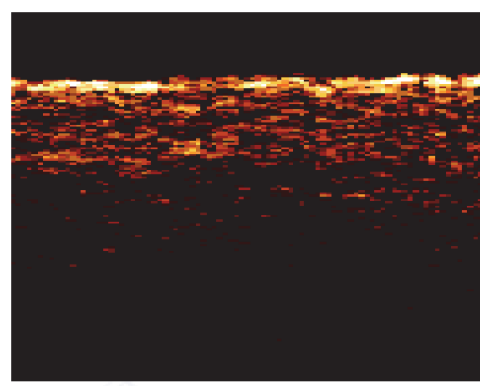

(e)

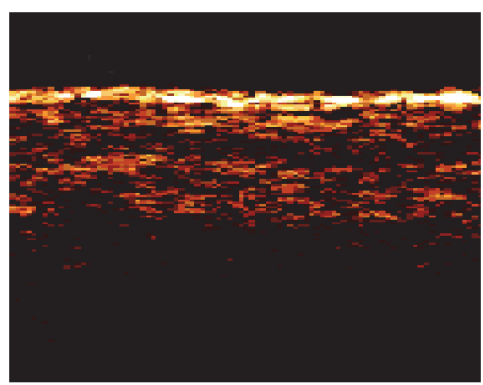

(c)

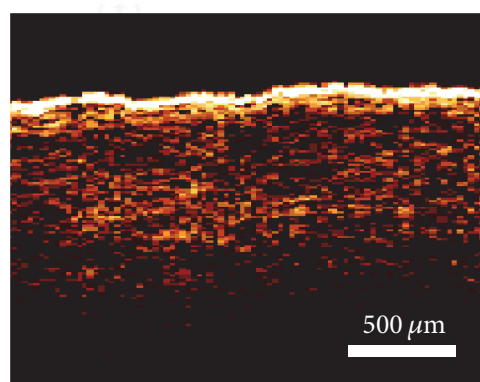

(f)

FIGURE 3: OCT images of the ankle of the right hind paws of the rats in the model group before modelling (a), 1 day after modelling (b), 8 days after modelling (c), 15 days after modelling (d), 22 days after modelling (e), and 29 days after modelling (f). The white scale in the image indicates the contrast scales.

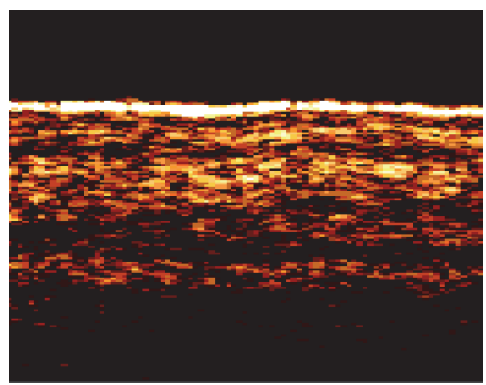

(a)

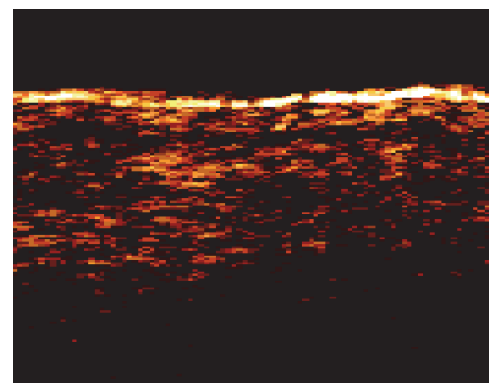

(d)

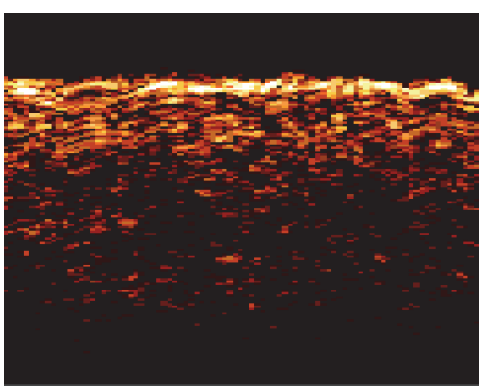

(b)

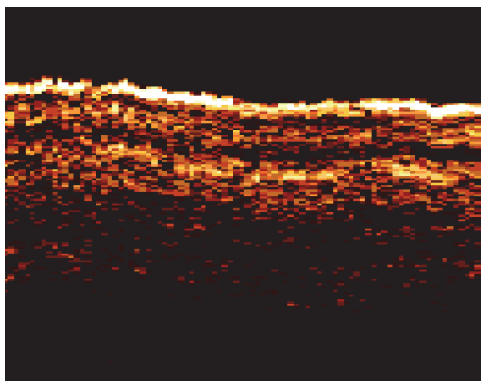

(e)

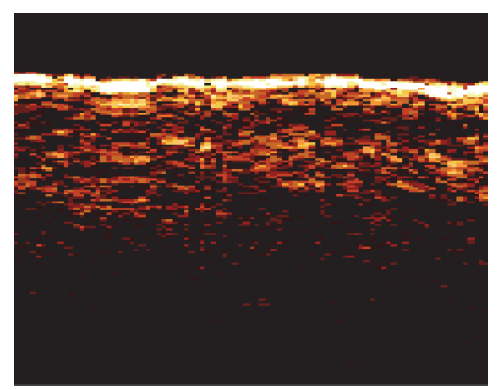

(c)

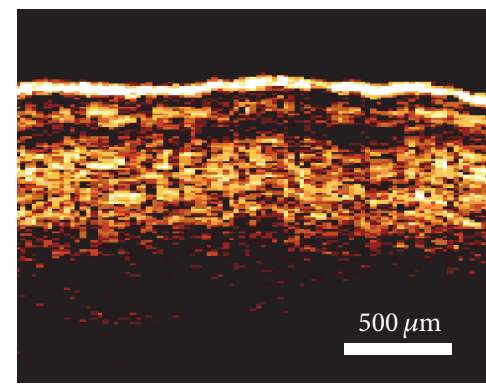

(f)

FIGURE 4: OCT images of the ankle of the right hind paws of the rats in the electroacupuncture group before modelling (a), 1 day after modelling (b), 8 days after modelling (c), 15 days after modelling (d), 22 days after modelling (e), and 29 days after modelling (f). The white scale in the image indicates the contrast scales. 


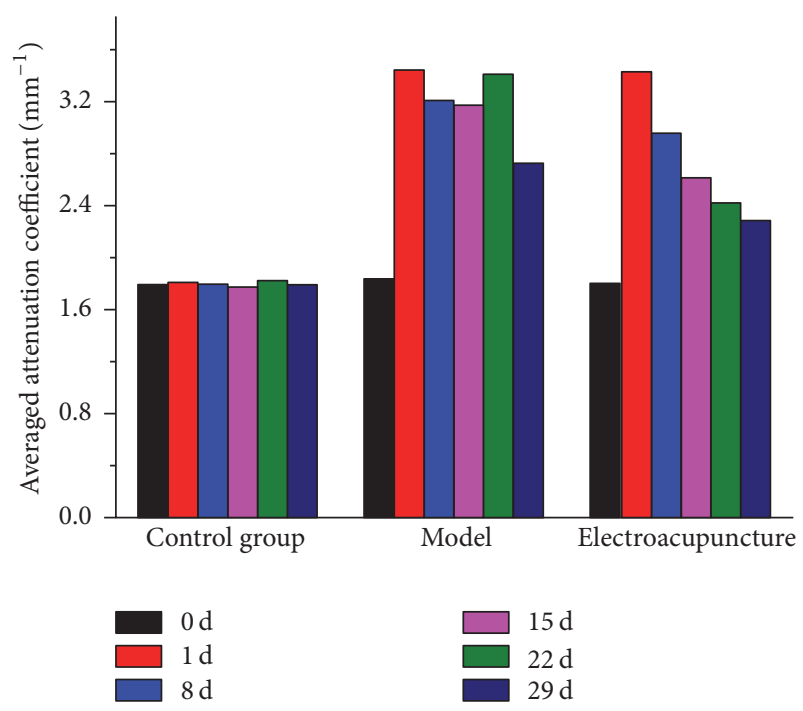

(a)

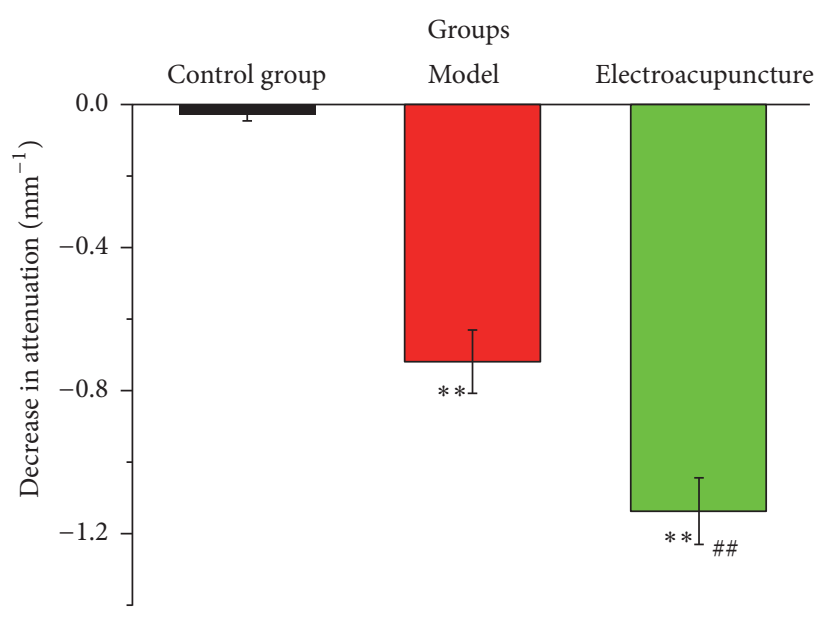

(b)

Figure 5: Averaged attenuation coefficients of the ankle of the right hind paws of the rats before modelling (0 day), 1 day after modelling (1st d), 8 days after modelling (8th d), 15 days after modelling (15th d), 22 days after modelling (22nd d), and 29 days after modelling (29th d) from each group (a). The decreased attenuation coefficient of the ankle of the right hind paws of the rats between on 29 th $d$ and the 1st $d$ in each group (control, model, and electroacupuncture groups); ${ }^{* *} P<0.01$ in comparison with control group; ${ }^{\# \#} P<0.01$ versus EA group at model group (b).

a clear structure is shown (Figure 4(a)). This condition indicated that the model was successfully established. In the electroacupuncture group, from the second day (1st d), the rats are treated with electroacupuncture in the ST36 acupoint at the right hind paw ipsilaterally. The structure becomes distinct with the treatment time increasing (Figures 4(c)-4(f)), as shown especially in Figure 4(f). This condition means that electroacupuncture can treat adjuvant-induced arthritis. In addition, the OCT can be used to detect the ankle in vivo.

The paper summarises the attenuation coefficients of the ankle of the right hind paws of the rats at $0,1,8,15,22$, and 29 days from the control, model, and electroacupuncture groups, respectively (Figure 5(a)). As seen from Figure 5(a), the light attenuation coefficients of the ankle in the control group did not change with the increase of the time. As also seen from Figure 5(a), comparing day 1 with day 0, the light attenuation coefficients of ankle tissue increased not only in the model group but also in the electroacupuncture group. This increase was observed as a high amount of light was absorbed after the model was established. The light attenuation coefficients of the ankle tissues decreased (e.g., 8 and 15 days) or increased (e.g., 22 days) in the model group because the rats in the model group were not treated after the model was established. In the electroacupuncture group, the light attenuation coefficients of ankle tissues decreased as the time was prolonged after electroacupuncture was administered (Figure 5(a)). These results agree with the obtained images, which showed that adjuvant-induced arthritis can be treated with electroacupuncture stimulating the "Zusanli" acupoint. There was decreased attenuation of the ankle of the right hind paws of the rats compared on the 29th day with the 1st day in the control, model, and electroacupuncture groups, respectively (Figure 5(b)). There was a significant difference in attenuation coefficient decrease between the 29th $\mathrm{d}$ and the 1 st d not only in electroacupuncture group compared with control group, but also in model group compared with control group $(P<0.01)$. It also could be found that there was a significant decrease in the electroacupuncture group compared with the model group (Figure 5(b)). Although the rats with adjuvant-induced arthritis could slowly reduce the swelling as autoimmunity, electroacupuncture group at "Zusanli" could better accelerate the healing of rats with rheumatoid arthritis (consistent with Figure 5 results). Acupuncture or electroacupuncture at "Zusanli" in rats with rheumatoid arthritis could significantly improve local and systemic symptoms, alleviate joint swelling, and prevent inflammatory reactions $[35,36]$. According to "Lingshu" (Miraculous Pivot), "If the syndrome of Zhuobi (fixed obstruction) does not disappear and its accompanying symptom of long coldness continues, the "Zusanli" (ST36) is chosen as the major acupoint to treat the syndrome."

\section{Conclusion}

Electroacupuncture could be employed to stimulate "Zusanli" acupoint and to accelerate treatment of rats with adjuvantinduced arthritis. This study also demonstrated that the OCT could be applied to monitor the treatment effect on the depth of the ankles of rats with adjuvant-induced arthritis in vivo. The treatment efficacy of drugs can also be determined by measuring the penetration depth of the ankles of rats through OCT or other strategies.

\section{Competing Interests}

The authors of this paper do not have any association with a commercial organisation or any financial interest in a product 
that may give rise to the perception of a potential conflict of interests.

\section{Acknowledgments}

This work is supported by the National Natural Science Foundation of China (61335011, 31300691, and 61275187), the Natural Science Foundation of Guangdong Province of China (2014A030311024 and 2014A030310306), the Science and Technology Project of Guangdong Province of China (2014A020212282 and 2012A080203008), Specialized Research Fund for the Doctoral Program of Higher Education of China (20114407110001 and 20134407120003), the Science and Technology Innovation Project of the Education Department of Guangdong Province of China (2013KJCX0052), and the Entrusted Technology Development Projects of Infinitus (China) Co., Ltd. (HPG/2013/11/1596).

\section{References}

[1] M. M. N. Wendt, A. B. de Sá-Nakanishi, C. V. de Castro Ghizoni et al., "Oxidative state and oxidative metabolism in the brain of rats with adjuvant-induced arthritis," Experimental and Molecular Pathology, vol. 98, no. 3, pp. 549-557, 2015.

[2] P. Totoson, K. Maguin-Gate, A. Monnier et al., "Etanercept improves endothelial function in Rat Adjuvant-Induced Arthritis: mechanisms involved," Fundamental \& Clinical Pharmacology, vol. 29, p. 2, 2015.

[3] D. Lorton, C. Lubahn, N. Klein, J. Schaller, and D. L. Bellinger, "Dual role for noradrenergic innervation of lymphoid tissue and arthritic joints in adjuvant-induced arthritis," Brain, Behavior, and Immunity, vol. 13, no. 4, pp. 315-334, 1999.

[4] B. Johnston, A. R. Burns, and P. Kubes, "A role for mast cells in the development of adjuvant-induced vasculitis and arthritis," The American Journal of Pathology, vol. 152, no. 2, pp. 555-563, 1998.

[5] H. Jawed, S. Anjum, S. I. Awan, and S. U. Simjee, "Antiarthritic effect of GN1, a novel synthetic analog of glucosamine, in the collagen-induced arthritis model in rats," Inflammation Research, vol. 60, no. 12, pp. 1113-1120, 2011.

[6] M. F. Rossato, C. Hoffmeister, R. Tonello, A. P. De Oliveira Ferreira, and J. Ferreira, "Anti-inflammatory effects of vitamin E on adjuvant-induced arthritis in rats," Inflammation, vol. 38, no. 2, pp. 606-615, 2015.

[7] L. Ganju, Y. Padwad, R. Singh et al., "Anti-inflammatory activity of Seabuckthorn (Hippophae rhamnoides) leaves," International Immunopharmacology, vol. 5, no. 12, pp. 1675-1684, 2005.

[8] J. Rovenský, K. Švík, M. Stančíková, R. Ištok, L. Ebringer, and M. Ferenčík, "Treatment of experimental adjuvant arthritis with the combination of methotrexate and lyophilized Enterococcus faecium enriched with organic selenium," Folia Microbiologica, vol. 47, no. 5, pp. 573-578, 2002.

[9] K. Schiene, J. De Vry, and T. M. Tzschentke, "Antinociceptive and antihyperalgesic effects of tapentadol in animal models of inflammatory pain," Journal of Pharmacology and Experimental Therapeutics, vol. 339, no. 2, pp. 537-544, 2011.

[10] J. Lin, H. Huang, G.-H. Ding, and D. Zhang, "Relationship between the function of mast cells and acupuncture analgesia in adjuvant arthritis rats," Zhen Ci Yan Jiu, vol. 32, no. 1, pp. 1619, 2007.
[11] D. Huang, E. A. Swanson, C. P. Lin et al., "Optical coherence tomography," Science, vol. 254, no. 5035, pp. 1178-1181, 1991.

[12] W. Drexler, U. Morgner, F. X. Kärtner et al., "In vivo ultrahighresolution optical coherence tomography," Optics Letters, vol. 24, no. 17, pp. 1221-1223, 1999.

[13] N. Pahuja, R. Shetty, C. Jayadev, R. Nuijts, B. Hedge, and V. Arora, "Intraoperative optical coherence tomography using the RESCAN 700: preliminary results in collagen crosslinking," BioMed Research International, vol. 2015, Article ID 572698, 7 pages, 2015.

[14] J. A. Izatt, M. R. Hee, E. A. Swanson et al., "Micrometer-scale resolution imaging of the anterior eye in vivo with optical coherence tomography," Archives of Ophthalmology, vol. 112, no. 12, pp. 1584-1589, 1994.

[15] M. R. Hee, J. A. Izatt, E. A. Swanson et al., "Optical coherence tomography of the human retina," Archives of Ophthalmology, vol. 113, no. 3, pp. 325-332, 1995.

[16] C. E. Saxer, J. F. De Boer, B. H. Park, Y. Zhao, Z. Chen, and J. S. Nelson, "High-speed fiber-based polarization-sensitive optical coherence tomography of in vivo human skin," Optics Letters, vol. 25, no. 18, pp. 1355-1357, 2000.

[17] J. Welzel, "Optical coherence tomography in dermatology: a review," Skin Research and Technology, vol. 7, no. 1, pp. 1-9, 2001.

[18] J. Welzel, E. Lankenau, R. Birngruber, and R. Engelhardt, "Optical coherence tomography of the human skin," Journal of the American Academy of Dermatology, vol. 37, no. 6, pp. 958963, 1997.

[19] E. Vaghefi and B. Pontré, "Application of arterial spin labelling in the assessment of ocular tissues," BioMed Research International, vol. 2016, Article ID 6240504, 13 pages, 2016.

[20] H. Ullah, G. Gilanie, F. Hussain, and E. Ahmad, "Autocorrelation optical coherence tomography for glucose quantification in blood," Laser Physics Letters, vol. 12, no. 12, Article ID 125602, 2015.

[21] A. Baumgartner, S. Dichtl, C. K. Hitzenberger et al., "Polarization-sensitive optical coherence tomography of dental structures," Caries Research, vol. 34, no. 1, pp. 59-69, 2000.

[22] F. I. Feldchtein, G. V. Gelikonov, V. M. Gelikonov et al., "In vivo OCT imaging of hard and soft tissue of the oral cavity," Optics Express, vol. 3, no. 6, pp. 239-250, 1998.

[23] M.-T. Tsai, H.-C. Lee, C.-K. Lee et al., "Effective indicators for diagnosis of oral cancer using optical coherence tomography," Optics Express, vol. 16, no. 20, pp. 15847-15862, 2008.

[24] P. Wilder-Smith, W.-G. Jung, M. Brenner et al., "In vivo optical coherence tomography for the diagnosis of oral malignancy," Lasers in Surgery and Medicine, vol. 35, no. 4, pp. 269-275, 2004.

[25] H. X. Dong, Z. Y. Guo, C. C. Zeng et al., "Quantitative analysis on tongue inspection in Traditional Chinese Medicine using optical coherence tomography," Journal of Biomedical Optics, vol. 13, no. 1, Article ID 011004, 2008.

[26] V. Erthal, M. D. Da Silva, F. J. Cidral-Filho, A. R. S. Santos, and P. Nohama, "ST36 laser acupuncture reduces pain-related behavior in rats: involvement of the opioidergic and serotonergic systems," Lasers in Medical Science, vol. 28, no. 5, pp. 13451351, 2013.

[27] T.-F. He, W.-J. Yang, S.-H. Zhang, C.-Y. Zhang, L.-B. Li, and Y.F. Chen, "Electroacupuncture inhibits inflammation reaction by upregulating vasoactive intestinal peptide in rats with adjuvantinduced arthritis," Evidence-Based Complementary and Alternative Medicine, vol. 2011, Article ID 290489, 8 pages, 2011. 
[28] Y. H. Baek, D. Y. Choi, H. I. Yang, and D. S. Park, "Analgesic effect of electroacupuncture on inflammatory pain in the rat model of collagen-induced arthritis: mediation by cholinergic and serotonergic receptors," Brain Research, vol. 1057, no. 1-2, pp. 181-185, 2005.

[29] R.-X. Zhang, L. X. Lao, X. Y. Wang et al., "Electroacupuncture attenuates inflammation in a rat model," Journal of Alternative and Complementary Medicine, vol. 11, no. 1, pp. 135-142, 2005.

[30] H. Zhong, Z. Guo, H. Wei et al., "In vitro study of ultrasound and different-concentration glycerol-induced changes in human skin optical attenuation assessed with optical coherence tomography," Journal of Biomedical Optics, vol. 15, no. 3, Article ID 036012, 2010.

[31] Z. Huiqing, G. Zhouyi, W. Huajiang et al., "Quantification of glycerol diffusion in human normal and cancer breast tissuesin vitrowith optical coherence tomography," Laser Physics Letters, vol. 7, no. 4, pp. 315-320, 2010.

[32] K. V. Larin, M. S. Eledrisi, M. Motamedi, and R. O. Esenaliev, "Noninvasive blood glucose monitoring with optical coherence tomography-a pilot study in human subjects," Diabetes Care, vol. 25, no. 12, pp. 2263-2267, 2002.

[33] K. V. Larin, M. Motamedi, T. V. Ashitkov, and R. O. Esenaliev, "Specificity of noninvasive blood glucose sensing using optical coherence tomography technique: A Pilot Study," Physics in Medicine and Biology, vol. 48, no. 10, pp. 1371-1390, 2003.

[34] H. Q. Zhong, Z. D. Zhang, Z. Y. Guo et al., "Using OCT image to distinguish human acupoint from non-acupoint tissues after irradiation with laser in vivo: a pilot study," Chinese Optics Letters, vol. 8, no. 4, pp. 418-420, 2010.

[35] Y. Jian, H. Ling, S. Xiao-Ge et al., "'Shènshù' (BL 23)and 'Zúsānlî' (ST 36) on Ras-MAPK signal pathways in synovial tissues of rats with experimental rheumatoid arthritis," World Journal of Acupuncture-Moxibustion, no. 2, pp. 29-33, 2013.

[36] D. S. Park, B. K. Seo, and Y. H. Baek, "Analgesic effect of electroacupuncture on inflammatory pain in collagen-induced arthritis rats: mediation by alpha2- and beta-adrenoceptors," Rheumatology International, vol. 33, no. 2, pp. 309-314, 2013. 

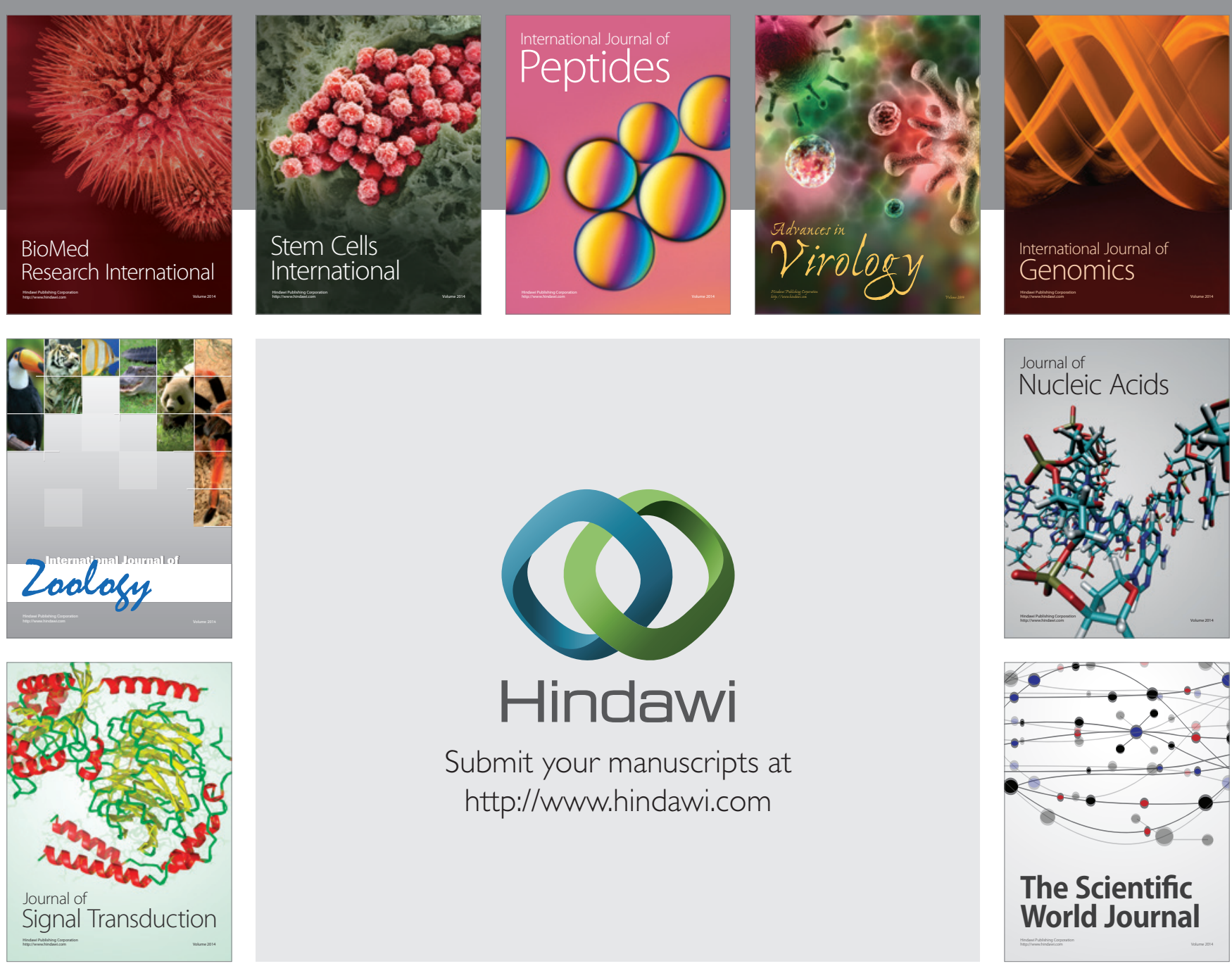

Submit your manuscripts at

http://www.hindawi.com
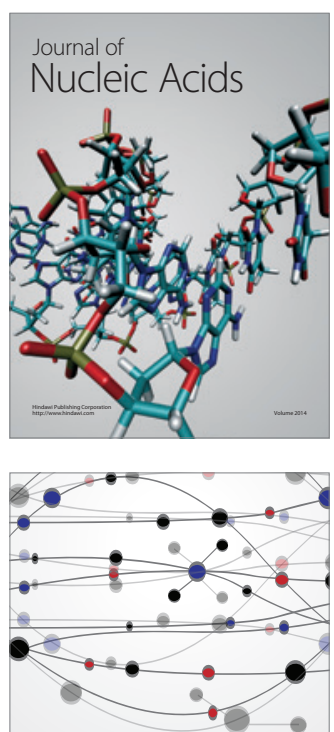

The Scientific World Journal
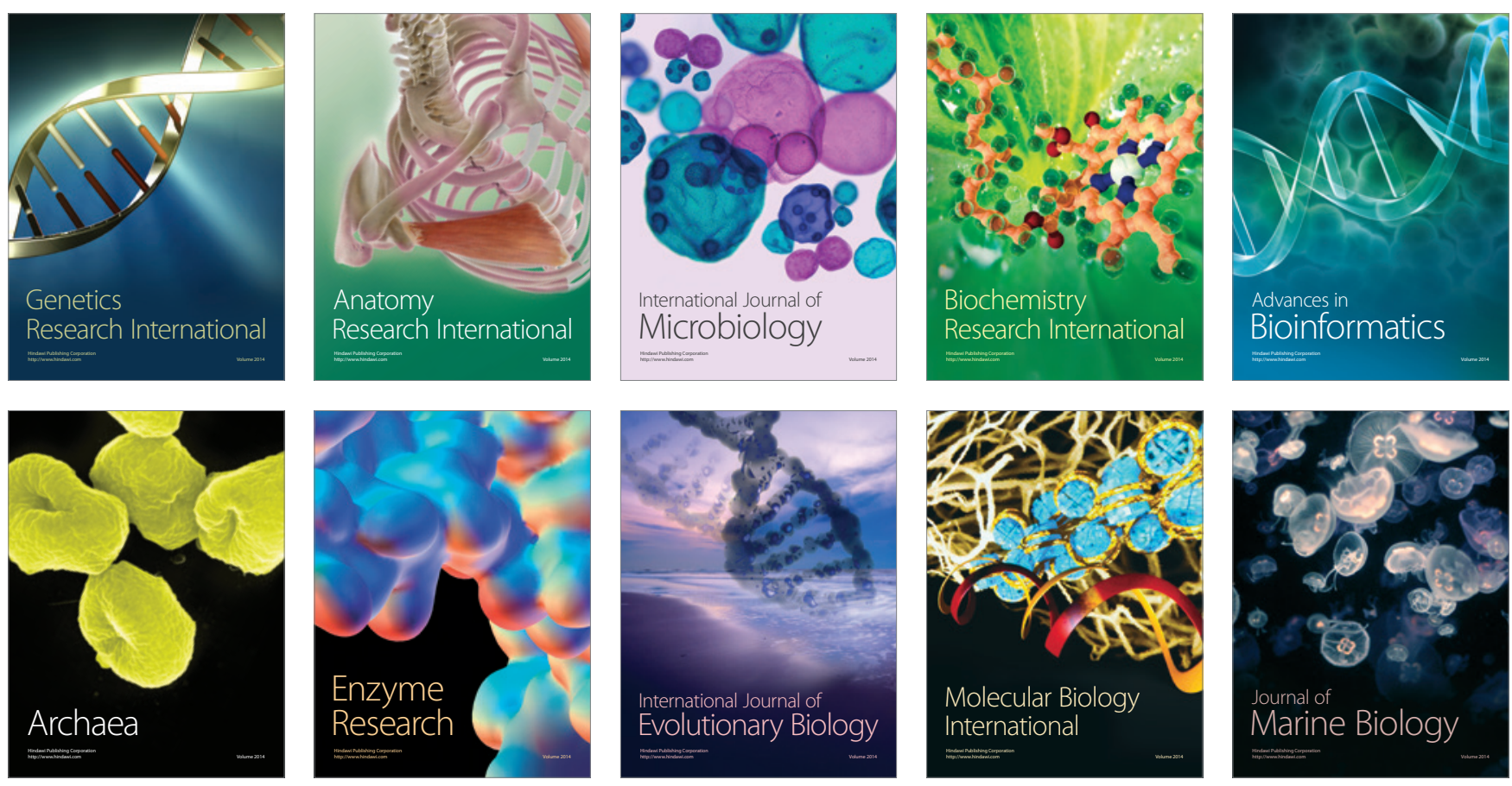